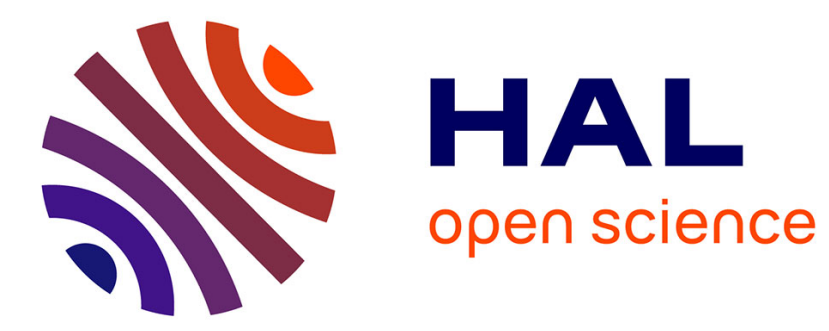

\title{
Acoustical and mechanical characterization of poroelastic materials using a Bayesian approach
}

Jean-Daniel Chazot, Erliang Zhang, Jérôme Antoni

\section{To cite this version:}

Jean-Daniel Chazot, Erliang Zhang, Jérôme Antoni. Acoustical and mechanical characterization of poroelastic materials using a Bayesian approach. Journal of the Acoustical Society of America, 2012, 131 (6), pp.4584-4595. hal-01018735

\section{HAL Id: hal-01018735 \\ https://hal.science/hal-01018735}

Submitted on 3 Sep 2021

HAL is a multi-disciplinary open access archive for the deposit and dissemination of scientific research documents, whether they are published or not. The documents may come from teaching and research institutions in France or abroad, or from public or private research centers.
L'archive ouverte pluridisciplinaire HAL, est destinée au dépôt et à la diffusion de documents scientifiques de niveau recherche, publiés ou non, émanant des établissements d'enseignement et de recherche français ou étrangers, des laboratoires publics ou privés. 


\title{
Acoustical and mechanical characterization of poroelastic materials using a Bayesian approach
}

\author{
Jean-Daniel Chazot $^{\text {a) }}$ and Erliang Zhang \\ Université de Technologie de Compiègne, CNRS UMR 6253 Roberval, Centre de Recherche de Royallieu, \\ BP 20529, 60205 Compiègne Cedex, France \\ Jérôme Antoni \\ Vibrations and Acoustic Laboratory, 25 bis avenue Jean Capelle, University of Lyon F-69621 Villeurbanne \\ Cédex, France
}

(Received 24 February 2011; revised 24 February 2012; accepted 9 March 2012)

\begin{abstract}
A characterization method of poroelastic materials saturated by air is described. This inverse method enables the evaluation of all the parameters with a simple measurement in a standing wave tube. Moreover, a Bayesian approach is used to return probabilistic data such as the maximum a posteriori and the confidence interval of each parameter. To get these data, it is necessary to define prior probability distributions on the parameters characterizing the studied material. This last point is very important to regularize the inverse problem of identification. In a first step, the direct problem formulation is presented. Then, the inverse characterization is developed and applied to simulated and experimental data. (C) 2012 Acoustical Society of America. [http://dx.doi.org/10.1121/1.3699236]
\end{abstract}

PACS number(s): 43.55.Ev, 43.20.Gp, 43.20.Jr, 43.20.Ye [FCS]

Pages: 4584-4595

\section{INTRODUCTION}

Porous materials are used in a wide range of applications such as automotive and aeronautics to improve the acoustic properties at mid and high frequencies without adding an excessive mass. The sound absorption realized with these materials is due to viscous and thermal dissipations inside the pores. Therefore, the structural microgeometry is of prime importance to describe these phenomena. To predict the behavior of porous materials, finite element models $^{1,2}$ (FEM) based on Biot's theory ${ }^{3-5}$ can be employed. Several parameters are then necessary for their description: the shear modulus $N$, the bulk modulus $K_{b}$, the structural damping $\eta_{s}$, the density of the solid frame $\rho_{1}$, the porosity $\phi$, the tortuosity $\alpha_{\infty}$, the airflow resistivity $\sigma$, and the viscous and thermal characteristic lengths $\Lambda$ and $\Lambda^{\prime}$. Three methods are typically available to evaluate these parameters: direct measurements, indirect measurements, and inverse measurements.

\section{A. Direct measurements}

All the parameters can be measured separately with specific apparatuses. A porosimeter is used to measure the porosity via a pressure variation technique. ${ }^{6}$ If the frame does not conduct electricity, the tortuosity can be measured with an electrical resistivity measurement. ${ }^{7}$ Otherwise, the tortuosity is obtained with ultrasonic wavespeed measurements in the porous material saturated by two different gases. ${ }^{8-10}$ This method returns also the viscous and thermal characteristic lengths. The airflow resistivity is measured with a flowmeter. ${ }^{11}$ Finally, elastic and damping parameters related to

\footnotetext{
a) Author to whom correspondence should be addressed. Electronic mail: jean-daniel.chazot@utc.fr
}

the solid phase can be measured with classical experimental methods adapted for porous materials. A review of these methods is found in Ref. 12, and an application to the characterization of glass wools is presented in Ref. 13. Direct measurements of all the parameters can also be performed by analyzing the microstructure of the frame with a threedimensional (3D) microtomograph. ${ }^{14,15}$

However, all these methods present several drawbacks and difficulties. The saturation of a porous material with a fluid is not always easy and may also damage the microstructure. Uncertainties related to ultrasonic wavespeed measurements can be important in some cases. The resistivity measurements are affected by the boundary conditions of the sample in the apparatus. And finally, some materials like fibrous ones can present a non-linear dynamical behavior. The parameters then depend on the compressional rate or on the strain level at which the sample is tested. ${ }^{16,17}$

\section{B. Indirect measurements}

Indirect measurements of the tortuosity and the viscous and thermal characteristic lengths are presented in Refs. 18 and 19. These parameters are extracted from pressure measurements in an impedance tube with an analytical solution. In the case of low or medium flow resistivity porous samples, the static airflow resistivity can also be extrapolated from these measurements. However, the values of the airflow resistivity and porosity are generally required as inputs and measured with a direct method. A similar analytical method based on ultrasonic measurements was developed in Ref. 20 with the advantage of evaluating the porosity at the same time. The drawback in this method is that its accuracy depends on the pressure measurements, but also on the uncertainties on the input parameters measured directly. Finally, the solid phase parameters can also be measured 
indirectly by measuring the phase velocities of guided acoustic waves (see Refs. 21 and 22).

\section{Inverse measurements}

Inverse measurements presented, for example, in Refs. 23 and 24 differ from the previous indirect measurements by the fact that an optimization tool is necessary to adjust a model on measured data. The accuracy of the model is of prime importance in these measurements but the optimization tool efficiency is also essential to find the global optimum without being trapped in a local minimum. It is therefore theoretically possible to infer all the parameters at once with only one measurement. The standard intrinsic parameters of porous materials can hence be evaluated, ${ }^{25-27}$ as well as the elastic parameters such as the

shear modulus. ${ }^{28}$ Inverse methods based on ultrasonic measurements have also been developed in Refs. 29-32. Here, the accuracy of the estimated parameters depends on the quality of the measurements and on the robustness of the optimization method. However, the reliability of the results is always difficult to assess.

In this paper, a robust inverse method is presented to characterize all the acoustic and elastic parameters at once in a standing wave tube. In particular, the Bayesian approach is adopted as it can naturally merge the experimental and prior information (see Ref. 33). As such, the resulting cost function is a combination of a data-fitness metric and a regularization term; the importance of the latter, which pops up as a natural byproduct of the Bayesian approach, is fundamental to improve the efficiency of the identification scheme especially in nearly non-identifiable configurations. Point estimates of the parameters are obtained by selecting the best samples returned by the Markov Chain Monte Carlo (MCMC) methods (i.e., samples after convergence of the chains). One of the most interesting features of the Bayesian approach in conjunction with MCMC method is to get the probability density function (pdf) of each parameter as well as the joint probability density functions of the parameters. Uncertainties can hence be quantified on estimated values of each parameter as well as dependencies between them. Having such information available is crucial to assess the quality of the parameters estimated from inverse measurements. Another advantage of the Bayesian approach exploited in this paper is its ability to include results from other direct or indirect measurements; the philosophy is then to view the latter as possible prior outcome.

The paper is organized as follows. First, the model used to describe the wave propagation in the standing wave tube with a porous sample is presented. Then the Bayesian approach used to identify the porous material parameters is detailed with its particular cost function.

The chosen optimization method is then discussed. Simulated and experimental results are finally presented.

\section{POROELASTIC MODEL DESCRIPTION}

The Biot's model is used here to calculate the reflexion and transmission coefficients of a porous material sample in a standing wave tube. Knowing these coefficients, it is possible to predict the pressure at any position inside the tube.

\section{A. Biot's model}

Poroelastic materials are defined in Biot's theory as materials with a fluid and a solid phase. Elastic, inertial, viscous, and thermal interactions between the two phases are hence taken into account. The elastic coupling is given by the Biot's poroelastic stress-strain relations

$$
\begin{aligned}
& \boldsymbol{\sigma}^{s}=2 N \boldsymbol{\varepsilon}^{s}+(P-2 N) \operatorname{tr}\left(\boldsymbol{\varepsilon}^{s}\right) \mathbf{I}+Q \operatorname{tr}\left(\boldsymbol{\varepsilon}^{f}\right) \mathbf{I}, \\
& \boldsymbol{\sigma}^{s}=R \operatorname{tr}\left(\boldsymbol{\varepsilon}^{f}\right) \mathbf{I}+Q \operatorname{tr}\left(\boldsymbol{\varepsilon}^{s}\right) \mathbf{I},
\end{aligned}
$$

where $\boldsymbol{\sigma}^{s}$ (respectively, $\boldsymbol{\sigma}^{f}$ ) represents the solid (respectively, fluid) stress tensor, and $\boldsymbol{\varepsilon}^{s}$ (respectively, $\boldsymbol{\varepsilon}^{f}$ ) represents the solid (respectively, fluid) strain tensor. I is the identity matrix, and $P, Q, R, N$ are the classical elastic coefficients used in Biot's model such as

$$
\begin{aligned}
& P=\frac{4}{3} N+K_{b}+\frac{(1-\phi)^{2}}{\phi} K_{f}, \\
& Q=\frac{R(1-\phi)}{\phi}, \\
& R=\phi K_{f},
\end{aligned}
$$

$N$ is the shear modulus and $K_{b}$ is the bulk modulus of the solid frame. $\phi$ is the porosity and $K_{f}$ is the fluid compressibility modulus. The solid phase is now defined with an elastic parameter $P_{e}$ and a structural damping $\eta_{s}$, independent from the fluid phase such that

$$
P=P_{e}\left(1-\mathrm{j} \eta_{s}\right)+\frac{(1-\phi)^{2}}{\phi} K_{f}
$$

On the other hand, the inertial coupling is taken into account in the equations of motion

$$
\begin{aligned}
& \nabla \sigma^{s}=-\rho_{11} \omega^{2} \mathbf{U}_{s}-\rho_{12} \omega^{2} \mathbf{U}_{f}, \\
& \nabla \sigma^{f}=-\rho_{22} \omega^{2} \mathbf{U}_{f}-\rho_{12} \omega^{2} \mathbf{U}_{s},
\end{aligned}
$$

where $\mathbf{U}_{s}$ and $\mathbf{U}_{f}$ are the solid and fluid displacements, and $\rho_{11}, \rho_{22}, \rho_{12}$ are the classical inertial coupling coefficients used in Biot's model and related to the densities $\rho_{1}$ of the solid phase, $\rho_{f}$ of the fluid phase, and $\rho_{0}$ of the air such that

$$
\begin{aligned}
& \rho_{11}=\rho_{1}+\phi \rho_{f}-\phi \rho_{0}, \\
& \rho_{12}=\phi \rho_{f}+\phi \rho_{0}, \\
& \rho_{22}=\phi \rho_{f} .
\end{aligned}
$$

Viscous and thermal dissipations are taken into account by the frequency-dependent Johnson-Allard's expressions of the fluid density $\rho_{f}$ and the dynamic fluid compressibility $K_{f}$ 


$$
\begin{gathered}
\rho_{f}=\rho_{0} \alpha_{\infty}\left(1-\frac{\sigma \phi}{\mathrm{j} \rho_{0} \alpha_{\infty} \omega} \sqrt{1-4 \mathrm{j} \frac{\eta \alpha_{\infty}^{2} \omega \rho_{0}}{\Lambda^{2} \phi^{2} \sigma^{2}}}\right) . \\
K_{f}=\frac{\gamma P_{0}}{\gamma-(\gamma-1)\left(1-\frac{8 \eta \sqrt{1-\mathrm{j} \rho_{0} \frac{\operatorname{Pr} \Lambda^{\prime 2} \omega}{16 \eta}}}{\mathrm{j} \Lambda^{\prime 2} \operatorname{Pr} \omega \rho_{0}}\right)^{-1}},
\end{gathered}
$$

respectively, with $\mathrm{j}^{2}=-1$. These expressions are given with a time dependence $e^{-\mathrm{j} \omega t}$, the Prandtl number Pr, the dynamic fluid viscosity $\eta$, the static pressure $P_{0}$, the ratio of specific heats $\gamma$, and five parameters: the porosity $\phi$, the tortuosity $\alpha_{\infty}$, the airflow resistivity $\sigma$, and the viscous and thermal characteristic lengths $\Lambda$ and $\Lambda^{\prime}$. In the following the ratio $k_{\Lambda}=\Lambda^{\prime} / \Lambda \geq 1$ is introduced in order to add the physical constraint $\Lambda^{\prime} \geq \Lambda$.

\section{B. Standing wave tube solution}

The case of a poroelastic material sample placed in a standing wave tube and submitted to a normal incident plane wave is considered as depicted in Fig. 1 (see also Ref. 16). The reduced one-dimensional Biot's model is therefore adopted. In this case, the shear wave is not present in the poroelastic material. Only the solid and fluid compressional waves remain. Using the wave formalism with a time dependence $e^{-\mathrm{j} \omega t}$, the fluid and solid displacements are derived from two scalar potentials

$$
\begin{aligned}
& \mathbf{U}_{s}=\nabla\left(\varphi_{1}+\varphi_{2}\right), \\
& \mathbf{U}_{s}=\nabla\left(\mu_{1} \varphi_{1}+\mu_{2} \varphi_{2}\right),
\end{aligned}
$$

with

$$
\begin{aligned}
& \varphi_{1}=A e^{\mathrm{j} k_{1} x}+B e^{-\mathrm{j} k_{1} x}, \\
& \varphi_{2}=C e^{\mathrm{j} k_{2} x}+D e^{-\mathrm{j} k_{2} x} .
\end{aligned}
$$

Wave numbers $k_{1}, k_{2}$ and amplitude coefficients $\mu_{1}, \mu_{2}$ between the solid and fluid displacements are recalled in the Appendix.

Unknown amplitudes $A, B, C$, and $D$ are determined using the boundary conditions at the interface of the poroelastic material sample. In the present case of an acoustic-poroelastic interface, the coupling conditions are based on the flow, the fluid pressure, and the total normal stress continuity conditions such that

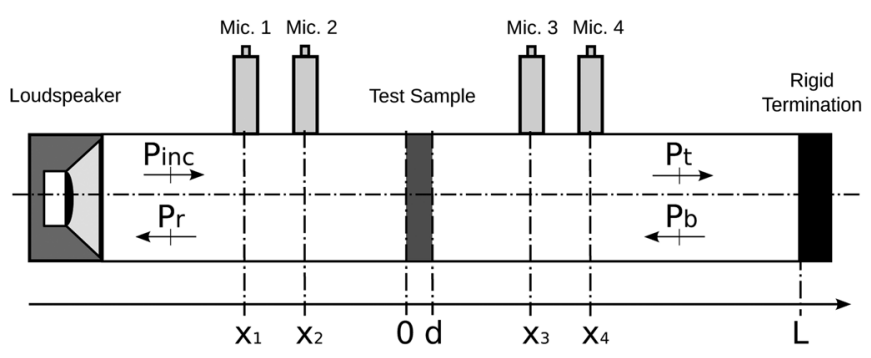

FIG. 1. Standing-wave tube setup.

$$
\begin{aligned}
& \phi \mathbf{U}_{f} \cdot \mathrm{n}+(1-\phi) \mathbf{U}_{s} \cdot \mathrm{n}=\mathbf{U}_{a} \cdot \mathrm{n} \\
& \phi \boldsymbol{\sigma}^{f} \cdot \mathrm{n}+(1-\phi) \boldsymbol{\sigma}^{s} \cdot \mathrm{n}=-P_{a} \cdot \mathrm{n} \\
& \boldsymbol{\sigma}^{f} \cdot \mathrm{n}=-P_{a} \cdot \mathrm{n} .
\end{aligned}
$$

The acoustic pressure $P_{a}$ and acoustic displacement $\mathbf{U}_{a}$ are related to the incident and reflected waves as

$$
P_{a}=P_{\text {inc }}+P_{r} \text { at } x=0,
$$

and to the transmitted and backward waves as

$$
P_{a}=P_{t}+P_{b} \text { at } x=d \text {. }
$$

On the other hand, reflected and transmitted waves are related to the incident wave with the reflection and transmission coefficients, respectively, such that

$$
\begin{aligned}
& \left\|P_{r}\right\|=\mathcal{R}\left\|P_{i n c}\right\|, \\
& \left\|P_{t}\right\|=\mathcal{T}\left\|P_{i n c}\right\|,
\end{aligned}
$$

while the backward wave is related to the transmitted wave with the rigid boundary condition as

$$
\mathbf{U}_{t}+\mathbf{U}_{b}=0 \text { at } x=L \text {. }
$$

It is thus possible to calculate the reflection and transmission coefficient of the porous material sample. Then the total acoustic pressure can be determined at any position in the tube, and, in particular, at the four microphone positions by summing the incident and reflected waves in the upstream section and by summing the transmitted and the backward waves in the downstream section. The only remaining unknown to set is the incident pressure.

\section{BAYESIAN IDENTIFICATION METHOD}

The central idea beyond the Bayesian approach is to construct the posterior pdf of the parameters to be inferred. Not only will the maximization of the latter provide the most likely values of the parameters given the measured datathe so-called maximum a posteriori (MAP) estimates-but its shape will be truly indicative of the joint probability distribution of the estimated parameters as well. ${ }^{33,34}$ In particular, it will give access to the full covariance matrix, a fundamental quantity to assess the variability of the estimates and their mutual correlations. This is in contrast to most other optimization techniques, where cost functions rarely result from a deductive approach and hardly bear any probabilistic interpretation.

\section{A. Cost function}

The parameters to identify are the following: the porosity $\phi$, the tortuosity $\alpha_{\infty}$, the airflow resistivity $\sigma$, the viscous characteristic length $\Lambda$, the characteristic lengths ratio $k_{\Lambda}$, the solid frame density $\rho_{1}$, the elastic part $P_{e}$ of the coefficient $P$ in Eq. (6), and the structural damping $\eta_{s}$. Namely, let $\theta=\left\{\phi, \alpha_{\infty}, \sigma, \Lambda, k_{\Lambda}, \rho_{1}, P_{e}, \eta_{s}\right\}$ be the vector of parameters 
to be inferred and $p\left(\theta \mid\left\{P_{i k}, P_{k}^{i n c}\right\}\right)$ its pdf conditional to the observations of the four pressures $P_{i k} \equiv P_{i}\left(\omega_{k}\right), i=1,2,3,4$, as returned by the microphones and the incident pressure $P_{k}^{i n c} \equiv P_{i n c}\left(\omega_{k}\right)$ at frequencies $\omega_{k}, k \in \mathcal{F}$. This is the posterior pdf which reflects all the information that can be inferred on $\theta$ from the measured data and in the frequency band $\left\{\omega_{k}, k \in \mathcal{F}\right\}$ based on the prior information. Now from Bayes' rule

$$
p\left(\theta \mid\left\{P_{i k}, P_{k}^{i n c}\right\}\right) \propto p\left(\left\{P_{i k}\right\} \mid \theta,\left\{P_{k}^{i n c}\right\}\right) p(\theta),
$$

where $\propto$ stands for the proportional sign [all factors entering Eq. (26) that do not depend on the vector of parameters $\theta$ have been removed], $p\left(\left\{P_{i k}\right\} \mid \theta,\left\{P_{k}^{i n c}\right\}\right)$ is the likelihood and $p(\theta)$ is the prior pdf of the parameters, both of which can be given closed-form expressions. In words, $p\left(\left\{P_{i k}\right\} \mid \theta,\left\{P_{k}^{i n c}\right\}\right)$ reflects the direct problem which, given the values of $\theta$ and $\left\{P_{\text {inc }}\left(\omega_{k}\right)\right\}$ can predict the data $\left\{P_{i}\left(\omega_{k}\right)\right\}$-notwithstanding measurement noise and modeling errors-whereas $p(\theta)$ is the mechanism to assign weights to the values of $\theta$ before the experiment. Typically, $p(\theta)$ will cover a range of possible values obtained from tabulated data or from other types of experiments (e.g., direct or indirect measurements) and its shape will be based either on the user's expertise or subjective judgment, or on strict physical constraints (e.g., constraint of positiveness). The choice of the prior pdf will be discussed in Sec. III B. That of the likelihood proceeds as follows.

As seen from Eqs. (21)-(24), the measured data are functionally related to the vector of parameters $\theta$ as

$$
P_{i k}=\beta_{i k}(\theta) P_{k}^{i n c}+N_{i k},
$$

where $\beta_{i k}(\theta)$ is a deterministic function that embodies the direct model of Sec. II, and where $N_{i k}$ accounts for additive measurement noise. It results that $p\left(\left\{P_{i k}\right\} \mid \theta,\left\{P_{k}^{i n c}\right\}\right)$ $=p_{N}\left(P_{i k}-\beta_{i k}(\theta) P_{k}^{i n c}\right)$ with $p_{N}(\cdot)$ standing for the pdf of $N_{i k}$. Upon invoking the central limit theorem applied to the Fourier transform of $N_{i k}$, it happens that under mild conditions $p_{N}(\cdot)$ tends to a (complex) Gaussian distribution, independently of the original pdf of the additive noise in the time domain. ${ }^{35}$ Hence, after further assuming that the measurement noise is uncorrelated across the microphones, which is true under stationary regime ${ }^{35}$

$$
\begin{aligned}
p\left(\theta \mid\left\{P_{i k}, P_{k}^{i n c}\right\}\right) \propto & p(\theta) \prod_{\omega_{k} \in \mathcal{F}} \frac{1}{\pi^{4} \prod_{i=1}^{4} \sigma_{i k}^{2}} \\
& \times \exp \left(-\sum_{i=1}^{4} \frac{\left|P_{i k}-\beta_{i k}(\theta) P_{k}^{i n c}\right|^{2}}{\sigma_{i k}^{2}}\right) .
\end{aligned}
$$

This is the closed-form expression of the posterior pdf of the parameters $\theta$ which can now be explored in a variety of ways. The noise variance $\sigma_{i k}^{2}$ enables here to increase or decrease the prior contribution in the posterior pdf. It may be determined either experimentally or set a priori. In the following, the variance is set to $0.5 \%$.

In the present work, the optimal values of the parameters are sought so as to maximize this expression or, more conveniently, so as to minimize its negative logarithm leading to the following cost function:

$$
J(\theta)=-\ln p(\theta)+\sum_{\omega_{k} \in \mathcal{F}} \sum_{i=1}^{4} \frac{\left|P_{i k}-\beta_{i k}(\theta) P_{k}^{i n c}\right|^{2}}{\sigma_{i k}^{2}} .
$$

In doing so, one should keep in mind that $P_{k}^{i n c}$ is the first quantity to be inferred since it is not measured by the microphones. This is easily achieved analytically by setting the gradient of $J(\theta)$ with respect to $P_{k}^{i n c}$ to zero, thus giving

$$
\hat{P}_{k}^{i n c}=\frac{\sum_{i=1}^{4} \beta_{i k}(\theta) * P_{i k}}{\sum_{i=1}^{4}\left|\beta_{i k}(\theta)\right|^{2}}
$$

(where * stands for the conjugate operator, and the hat sign indicates an estimate) which is to be used in place of $P_{k}^{i n c}$ in $J(\theta)$.

\section{B. Prior information}

The prior density function $p(\theta)$ on the inferred parameters is one of the most important quantities in the Bayesian approach. It is actually the mechanism by which the inverse problem is regularized, i.e., forced to find a unique and stable solution even in hardly identifiable cases. As mentioned above, there are many possible choices on $p(\theta)$ that may reflect the user's prior knowledge on the problem. In the present study, a separable pdf $p(\theta)=\Pi_{1}^{8} p\left(\theta_{i}\right)$ is chosen, meaning that the parameters are a priori mutually independent. It must be underlined here that prior independence only reflects the user's ignorance of any coupling between the parameters before the experience is conducted, and that it generally does not prevent the posterior parameters to be physically dependent, which after all is what matters only. Then, for the sake of simplicity, each pdf $p\left(\theta_{i}\right)$ was modeled as a Chi2 distribution with "scale parameter" $\lambda_{i}$ and number of degrees-of-freedom $v_{i}$, viz.,

$$
\begin{aligned}
\ln p\left(\theta_{i}\right)= & -\left(\lambda_{i}-1\right) \ln \left(a_{i} \theta_{i}+b_{i}\right) \\
& +\frac{\nu_{i}}{2}\left(a_{i} \theta_{i}+b_{i}\right)+C
\end{aligned}
$$

with $C$ a constant with no effect on $J(\theta)$. This naturally enforces a constraint of positivity $\left(a_{i}=1, b_{i}=0\right)$ on parameters $\sigma, \Lambda, \rho_{1}, P_{e}$, and $\eta_{s}$, and after a suitable change of variable $\left(a_{i}= \pm 1, b_{i}=\mp 1\right)$, inequality constraints $\phi \leq 1$, $\alpha_{\infty}>1$ and $k_{\Lambda} \geq 1$. In other words, it forbids non-physical values in Biot's model. Other distributions than the Chi2 could surely be used to ensure similar properties, yet it is well-known that the exact shape of the prior pdf has little effect when large amounts of data are available. ${ }^{34}$ Moreover, the values of $\lambda_{i}$ and $\nu_{i}$ may be tuned from their relations $\lambda_{i}=\mu_{\theta_{i}}^{2} / \sigma_{\theta_{i}}^{2}$ and $\nu_{i}=2 \mu_{\theta_{i}} / \sigma_{\theta_{i}}^{2}$ with the expected mean $\mu_{\theta_{i}}$ and variance $\sigma_{\theta_{i}}^{2}$ of the parameter $\theta_{i}$. Mean values and standard deviations used in the following are given in Table I for

TABLE I. Foam statistics.

\begin{tabular}{lcccccccc}
\hline \hline & $\phi$ & $\alpha_{\infty}$ & $\begin{array}{c}\sigma \\
\mathrm{kN} \mathrm{s} \mathrm{m}^{-4}\end{array}$ & $\begin{array}{c}\Lambda \\
\mu \mathrm{m}\end{array}$ & $k_{\Lambda}$ & $\begin{array}{c}\rho_{1} \\
\mathrm{~kg} \mathrm{~m}^{-3}\end{array}$ & $\begin{array}{c}P_{e} \\
\mathrm{MPa}\end{array}$ & $\eta_{s}$ \\
\hline Mean $\left(\mu_{\theta_{i}}\right)$ & 0.97 & 2.0 & 39 & 94 & 3.0 & 22 & 0.15 & 0.15 \\
St. Dev. $\left(\sigma_{\theta_{i}}\right)$ & 0.03 & 1.6 & 35 & 70 & 2.3 & 12 & 0.25 & 0.06 \\
\hline \hline
\end{tabular}


TABLE II. Fibrous statistics.

\begin{tabular}{lccccccccc}
\hline \hline & $\phi$ & $\alpha_{\infty}$ & $\begin{array}{c}\sigma \\
\mathrm{kN} \mathrm{s} \mathrm{m}^{-4}\end{array}$ & $\begin{array}{c}\Lambda \mathrm{m} \\
\mu\end{array}$ & $\begin{array}{c}k_{\Lambda} \\
\mathrm{kg} \mathrm{m}^{-3}\end{array}$ & $\begin{array}{c}P_{e} \\
\mathrm{MPa}\end{array}$ & $\eta_{s}$ \\
\hline Mean $\left(\mu_{\theta_{i}}\right)$ & 0.96 & 1.2 & 31 & 98 & 2.0 & 52 & 0.5 & 0.093 \\
Standard deviation $\left(\sigma_{\theta_{i}}\right)$ & 0.024 & 0.25 & 28 & 59 & 0.57 & 44 & 1.4 & 0.02 \\
\hline \hline
\end{tabular}

the foams, and in Table II for the fibrous materials. A good prior knowledge on some parameters is, of course, helpful to adjust these values. For example, a simple measurement with a balance can give a first estimation of the frame density in the void $\rho_{1}$. It must be emphasized here that although such prior information is crucial in the Bayesian approach, it could not be used in a classical approach, i.e., by direct plugin into Biot's model.

\section{OPTIMIZATION METHOD}

The aim of the optimization tools described here is to get a good estimation of each parameter in a robust way. Besides, the major problem to avoid is to be trapped in a local minimum of the cost function. With descent optimization methods, the result depends highly on the initial starting point. These methods are therefore generally used to refine locally a solution obtained from a global approach. However, global search methods like genetic algorithm or Monte Carlo methods are very time consuming when the number of parameters to estimate is important and when the search space is large.

MCMC techniques can serve to explore efficiently the whole space of the parameters when facing large dimensions. The basic idea is to generate random samples from a Markov Chain whose distribution converges to the posterior
TABLE III. Parameters of the three materials used in the simulated data.

\begin{tabular}{lccc}
\hline \hline Parameters & Material A & Material B & Material C \\
\hline$\phi$ & 0.95 & 0.95 & 0.97 \\
$\alpha_{\infty}$ & 1.00 & 1.00 & 1.54 \\
$\sigma\left(10^{3} \mathrm{~N} \mathrm{~m}^{-4} \mathrm{~s}\right)$ & 105 & 23.0 & 57.0 \\
$\Lambda(\mu \mathrm{m})$ & 35.1 & 54.1 & 24.6 \\
$k_{\Lambda}$ & 3 & 3 & 3 \\
$\rho_{1}\left(\mathrm{~kg} \mathrm{~m}^{-3}\right)$ & 17 & 58 & 46 \\
$P_{e}(\mathrm{MPa})$ & $1.4 \times 10^{-3}$ & $1.7 \times 10^{-2}$ & $2.9 \times 10^{-1}$ \\
$\eta_{s}$ & 0.100 & 0.100 & 0.115 \\
\hline \hline
\end{tabular}

pdf (28). A Markov chain (e.g., Metropolis-Hastings ${ }^{36,37}$ explores the search space by spending more time in regions of high probability, i.e., around the maximum of the cost function. Thus, optimal estimates of the parameters can be simply selected among samples of the Markov Chain at convergence. In addition, MCMC gives access to the joint pdf of the inferred parameters and is very useful to assess the accuracy of the parameters estimates. In cases where the parameters are hardly identifiable from the available measurements, the posterior pdf may indeed have multiple modes. As a result, there is a risk the Markov chain gets trapped in a local mode. To improve the exploration efficiency and avoid convergence to a local optimum, it has been proposed to combine a genetic algorithm with the MCMC method (see Refs. 38-40) - the so-called EMCMC method-where a series of chains are generated instead of a single one, and exchange of information is allowed between the chains through crossover and swap operators. The main drawback of this approach is the computation time necessary to ensure a good exploration when the search space is large, but this is not insurmountable, for instance, if a surrogate model is used. ${ }^{39}$
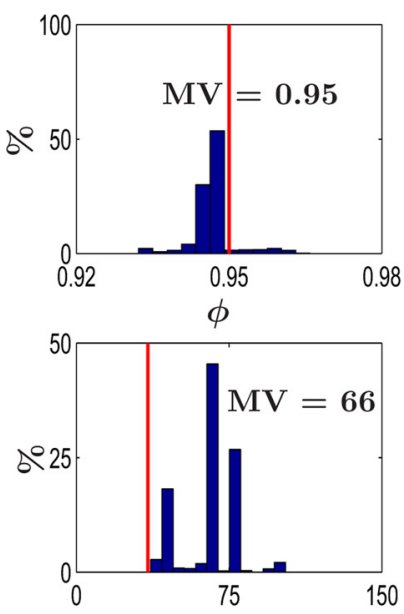

$\Lambda$

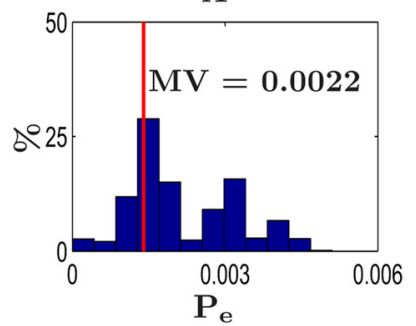

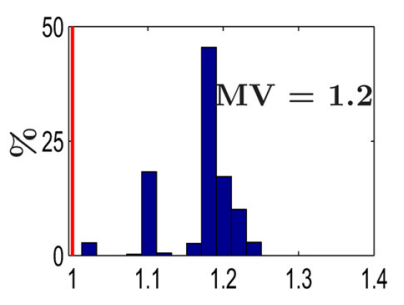
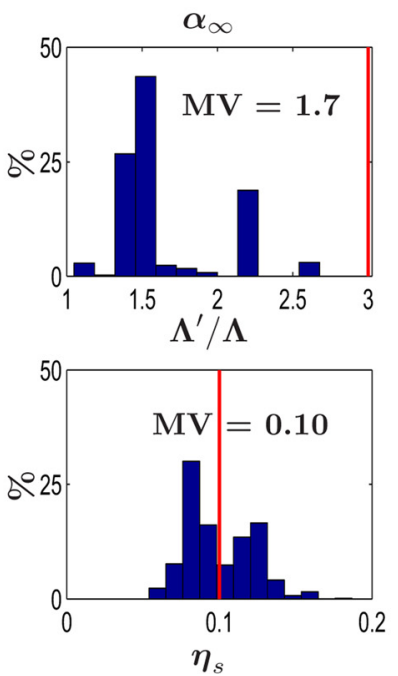
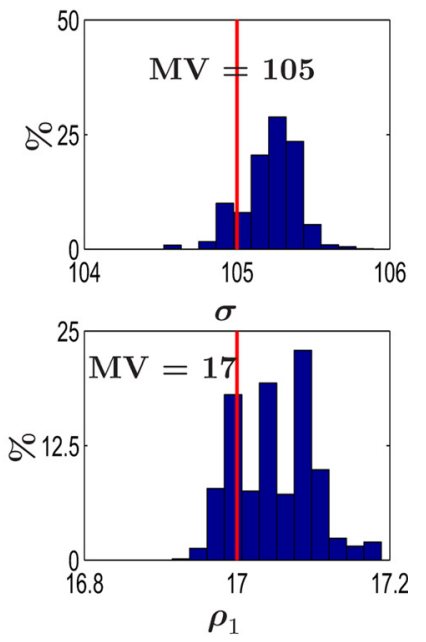

FIG. 2. (Color online) Posterior pdfs for the parameters of material A. Vertical line: reference value to find. 

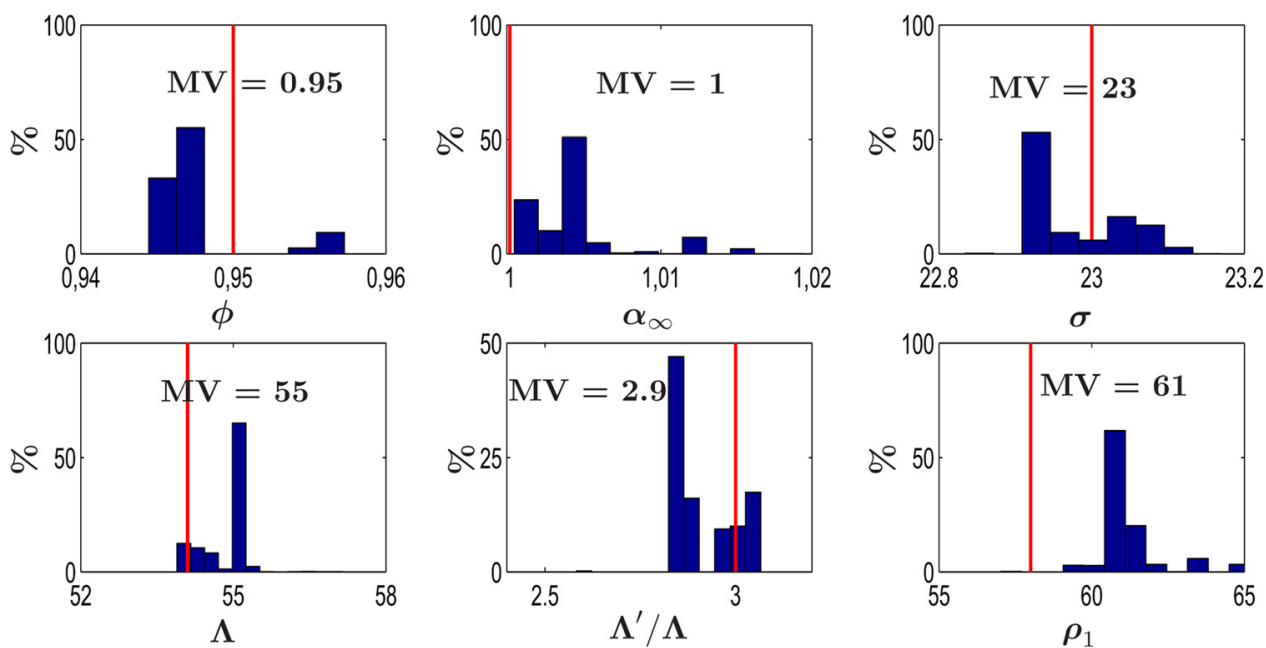

FIG. 3. (Color online) Posterior pdfs for the parameters of material B. Vertical line: reference value to find.
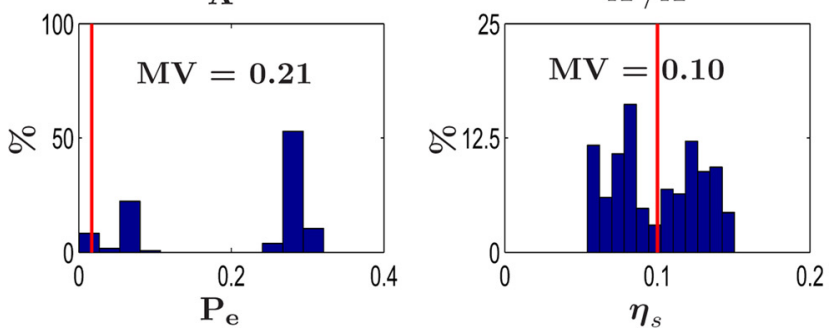

In the present work, 5000 generations with 240 individuals chains have been used to explore the parameters space. A serie of initial values has been generated randomly according to the prior pdfs - see Sec. III B-in order to ensure a good coverage of the search space. As previously explained, the prior information used in the cost function and detailed in Tables I and II makes it possible to restrict the search domain around the mean values of the parameters and within a "radius of convergence" proportional to their standard deviation.

\section{RESULTS}

\section{A. Results from simulated data}

In order to evaluate the efficiency and the robustness of the proposed identification method, simulated data are used first instead of real experimental measurements. These data are generated using the model described in Sec. II with 1181 frequencies lines between $100 \mathrm{~Hz}$ and $6000 \mathrm{~Hz}$. A random noise has been added to the simulated data with a signal-tonoise-ratio of $0.5 \%$ in order to account for instrumentation noise as well as modeling noise. The same noise variance $\sigma_{i k}^{2}$ is used in the cost function (29) with a direct effect on the prior contribution in the posterior pdf. When the level of noise is very low, the priors' contribution is not important. On the contrary, for high level of noise the estimated parameters depend highly on the prior contributions. In this case, the user expertise is of prime importance and estimated parameters must then be taken with caution. Three materials A, $\mathrm{B}$, and $\mathrm{C}$ found in Ref. 41 have hence been used with a sample thickness of $20 \mathrm{~mm}$. The parameters of each material are given in Table III. Material A is a low density glass wool found in aerospace applications with a very high airflow resistivity. Material B is a high density fibrous material and material $\mathrm{C}$ is a plastic foam with a stiff skeleton and a high

airflow resistivity. Both materials $\mathrm{B}$ and $\mathrm{C}$ are found in automotive applications.

The user's expertise and his/her subjective judgment is used here to modify the priors listed in Tables I and II. Material A being very soft and quite resistive, the statistical mean values of the rigidity and resistivity are multiplied by 0.01 and 3, respectively, to obtain the prior mean values. Material $\mathrm{B}$ being quite soft and not very resistive, the statistical mean values of the rigidity and resistivity are multiplied by 0.1 and $1 / 3$, respectively, to adjust efficiently the prior mean values.

The posterior pdfs and mean values (MVs) obtained with the EMCMC method for material A and B are presented in Figs. 2 and. 3, respectively. Most of the parameters are well identified with a narrow pdf around the reference values. However, it is seen that some parameters are not as accurately estimated as other ones. In particular, the tortuosity and the characteristic lengths of material A do not present a smooth distribution, but rather a multi-modal distribution. The mean value and the maximum a posteriori estimates are meaningless in this case, which demonstrates the advantage of returning the full pdfs rather than point estimates.

TABLE IV. Errors (\%) obtained with the simulated data.

\begin{tabular}{lccc}
\hline \hline Parameters & Material A & Material B & Material C \\
\hline$\phi$ & 0.33 & 0.24 & 0.97 \\
$\alpha_{\infty}$ & 17 & 0.44 & 4.8 \\
$\sigma$ & 0.21 & 0.10 & 2.8 \\
$\Lambda$ & 87 & 1.3 & 8.9 \\
$\Lambda^{\prime}$ & 3.3 & 1.6 & 4.2 \\
$\rho_{1}$ & 0.28 & 5.4 & 0.16 \\
$P_{e}$ & 56 & 1100 & 2.7 \\
$\eta_{s}$ & 0.32 & 0.07 & 16 \\
\hline \hline
\end{tabular}




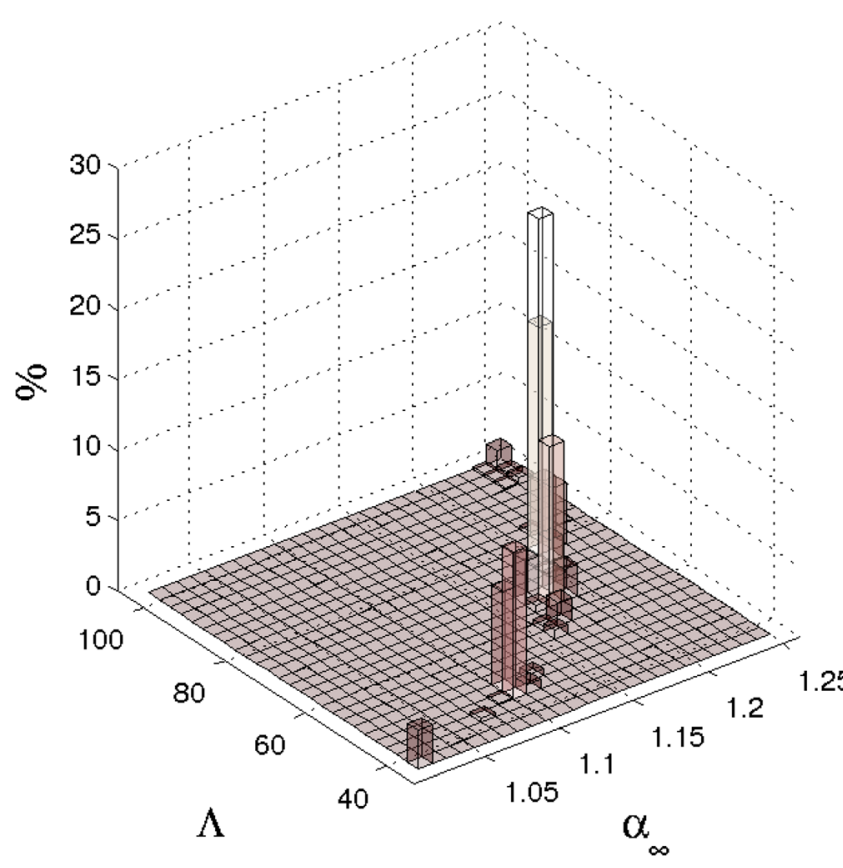

FIG. 4. (Color online) Joint pdf between the tortuosity and the viscous characteristic length for material $\mathrm{A}$.

Furthermore, these three parameters are not independent and the knowledge of one of them would enable one to estimate the two others more accurately.

Table IV displays the relative error obtained with the proposed method on simulated data with added random noise. The MVs are compared here with the reference data. It is seen that the best results are found with material $\mathrm{B}$, and the worst with material A. The results' accuracy can thus be related to the resistivity of the material. For material B, only the solid phase elasticity is not well-estimated. The solid phase is indeed not preponderant in the acoustic response due to the
TABLE V. Reference values for the three tested materials.

\begin{tabular}{lccc}
\hline \hline Parameters & Polyurethane foam & Fibrous material & Agglomerated \\
\hline$\phi$ & 0.98 & 0.96 & 0.91 \\
$\alpha_{\infty}$ & 1.74 & 1.00 & 1.09 \\
$\sigma\left(10^{3} \mathrm{~N} \mathrm{~m}^{-4} \mathrm{~s}\right)$ & 14.4 & 5.4 & 23.6 \\
$\Lambda(\mu \mathrm{m})$ & 87 & 170 & 33 \\
$k_{\Lambda}$ & 3.3 & 1.4 & 2.9 \\
$\rho_{1}\left(\mathrm{~kg} \mathrm{~m}^{-3}\right)$ & 28 & 44 & 120 \\
\hline \hline
\end{tabular}

acoustic excitation, the very low rigidity, and very low resistivity of the material. This is also visible on the pdf in Fig. 3. However, poor estimations of mechanical parameters do not affect the other recovered parameters. Indeed, when the solid phase contribution is negligible in the cost function, the solid phase parameters cannot be estimated accurately, yet it does not change the global optimum. For material A, the tortuosity and the viscous characteristic length are not accurate. However, when looking at the joint pdf in Fig. 4, one can see that these two parameters are closely related. A direct measurement of one of these parameters would therefore give a better estimation for the second. Here, again, this is a good demonstration of the advantage of getting the full posterior pdfs rather than only point estimates.

\section{B. Results from experimental data}

The identification process described in this paper relies on pressure measurements made in the standing wave tube of Fig. 1. A small or a large tube is used depending on the frequency band of interest. It is important here to reach high frequencies to get the effects of all the parameters including the solid phase elastic parameters. A multisine excitation is used with 1328 frequencies lines between $100 \mathrm{~Hz}$ and $6000 \mathrm{~Hz}$.
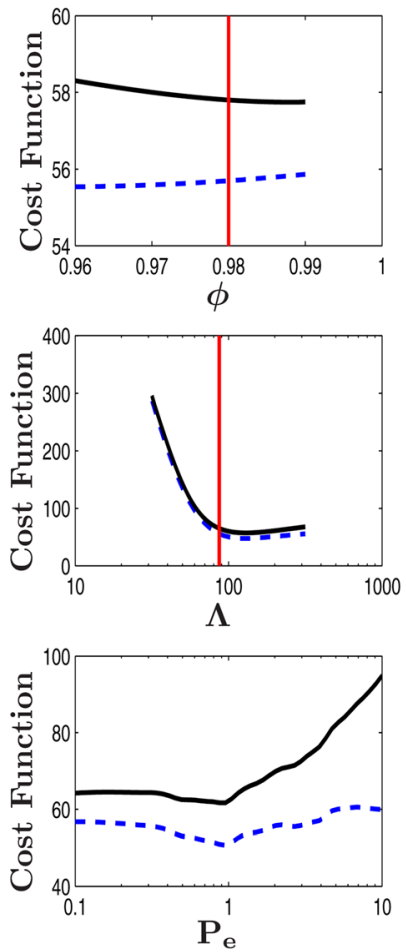
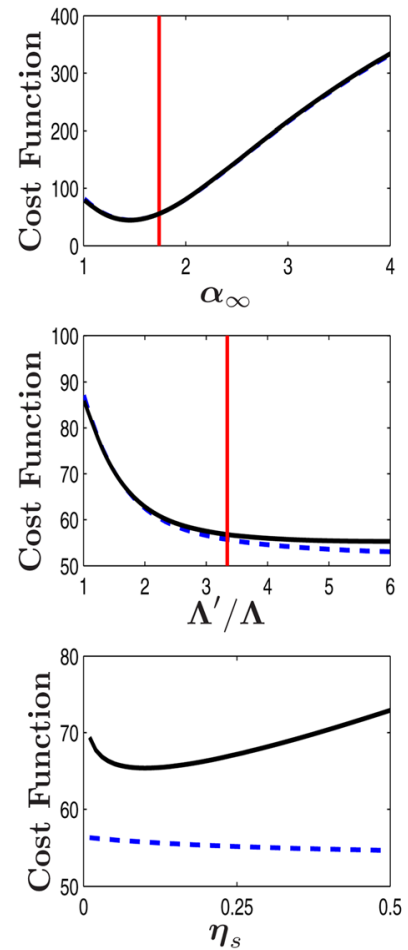
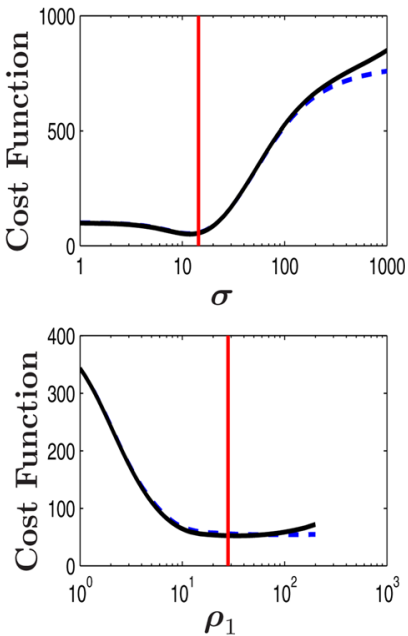

FIG. 5. (Color online) Cost function evaluation around the reference values for the foam. Solid lines: posterior cost functions, dotted lines: cost functions without prior information, vertical lines: reference values. 

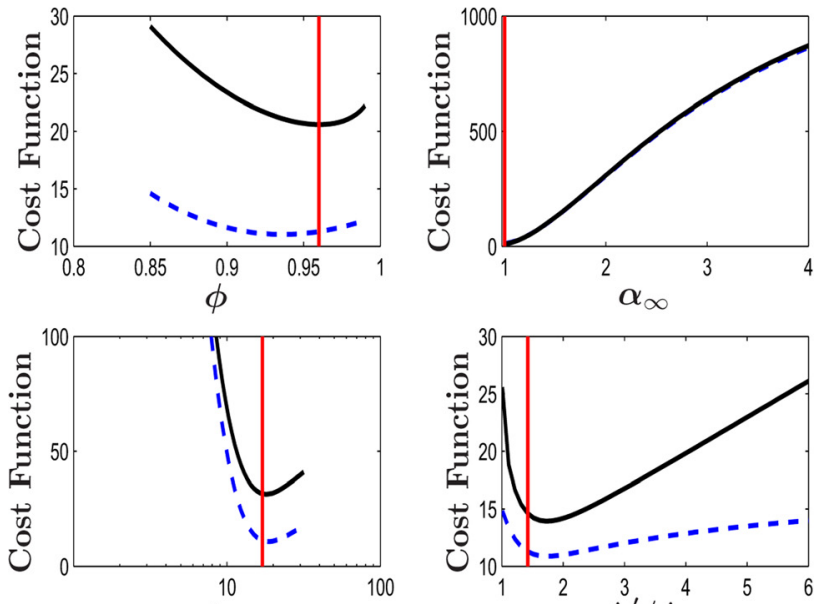

$\Lambda$
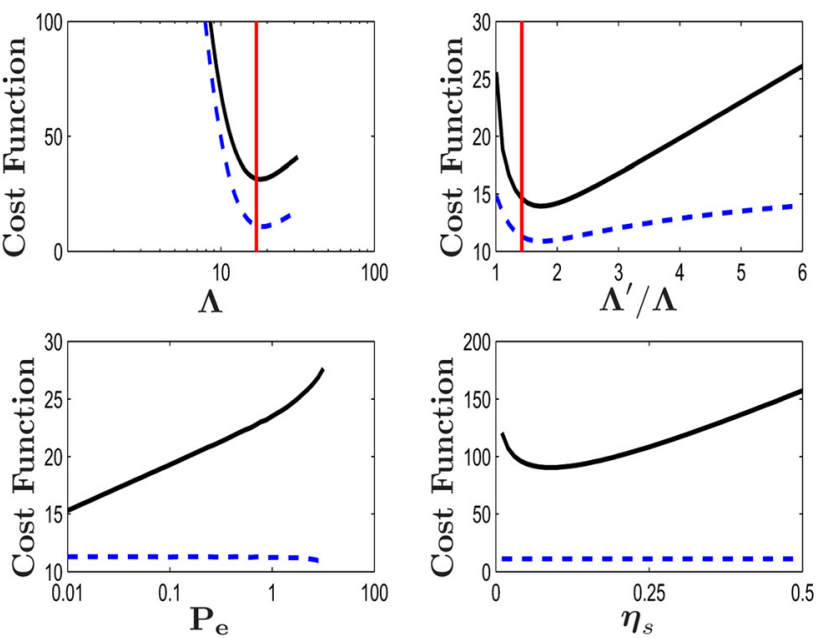

Three different materials are tested: a foam, a fibrous material, and an agglomerated foam material. The thickness of each sample is $4 \mathrm{~cm}, 4 \mathrm{~cm}$, and $2 \mathrm{~cm}$, respectively. The present method does not require several measurements on different samples for each material to get the pdfs of the identified parameters. This is another advantage of the Bayesian method: it takes into account both the measurement uncertainties and the modeling discrepancies at once with only one sample. Supplementary data on other samples could also be used to improve the identification
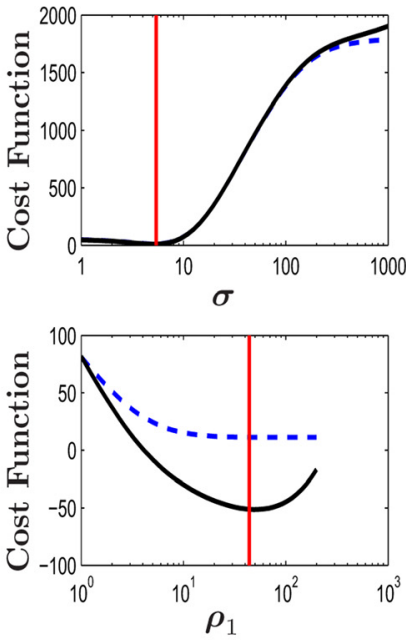

FIG. 6. (Color online) Cost function evaluation around the reference values for the fibrous material. Solid lines: posterior cost functions, dotted lines: cost functions without prior information, vertical lines: reference values.
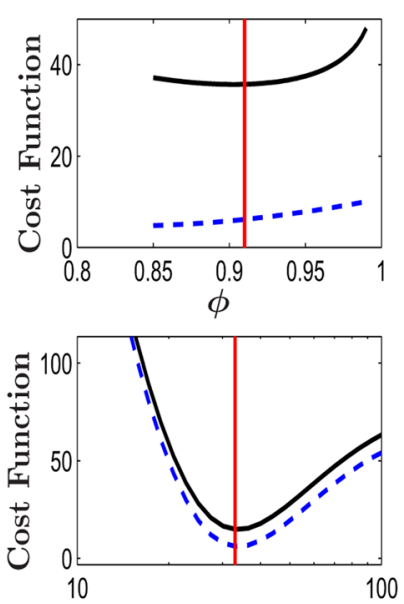

$\Lambda$
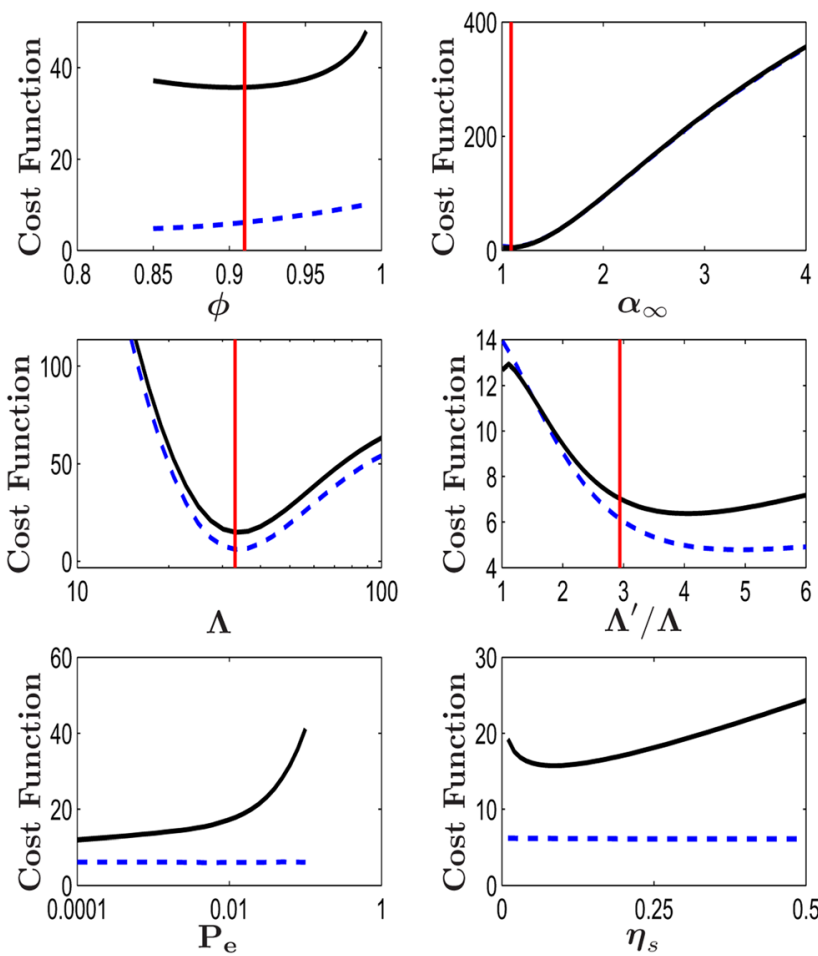

process, but is not necessary to get the posterior pdfs. The three materials have also been characterized by Matelys with the indirect method given in Refs. 18 and 19. These measured values are displayed in Table $\mathrm{V}$ and are taken in the following as reference values. However, these values are also submitted to some uncertainties inherent to the measurement setup or the boundary conditions of the sample, ${ }^{16,42}$ for instance.

For simplicity, the prior pdfs of the parameters are all defined by Chi2 pdfs-which easily allow non-negativity or
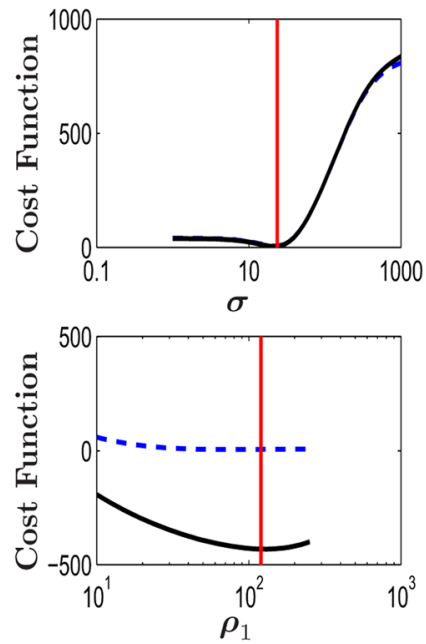

FIG. 7. (Color online) Cost function evaluation around the reference values for the agglomerated material. Solid lines: posterior cost functions, dotted lines: cost functions without prior information, vertical lines: reference values. 

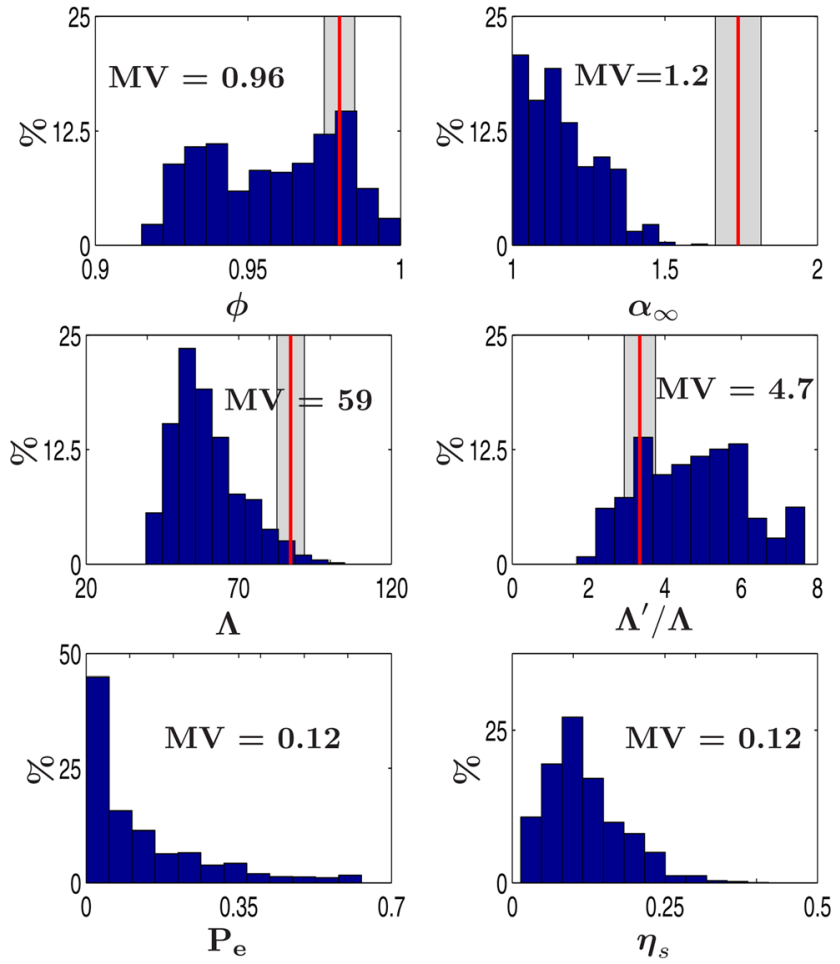

other inequality constraints-based on the tabulated data in Tables I and II. The user's expertise and subjective judgment are also used to amend the latter values, if necessary. For instance, the statistical mean value of the resistivity of the foam and the fibrous material have been multiplied by $1 / 3$ according to the authors' experience with this material. Similarly, the agglomerate material being denser, its porosity and density have been adjusted by multiplying their statistical mean values by 0.95 and 6 , respectively, to obtain the prior mean values.
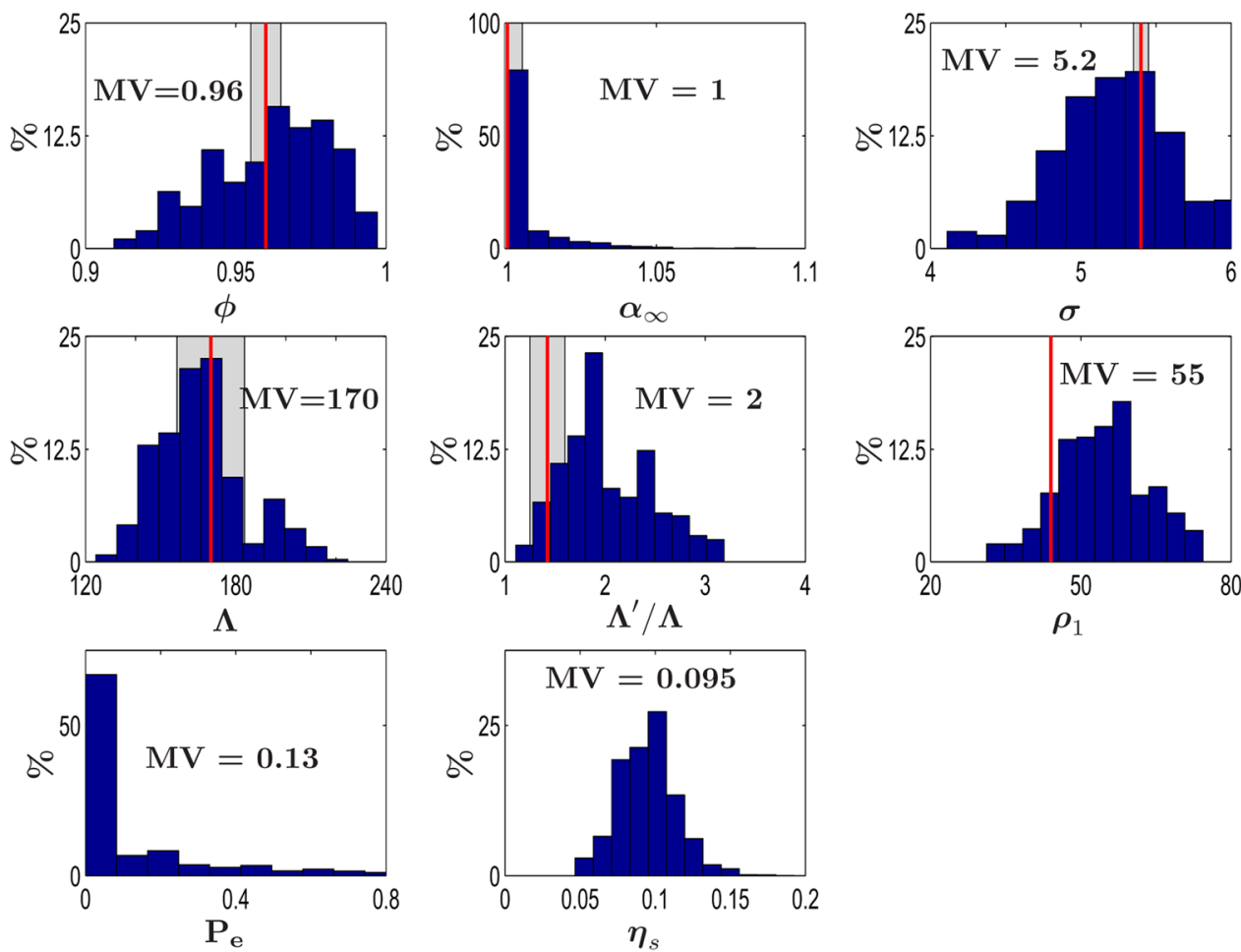
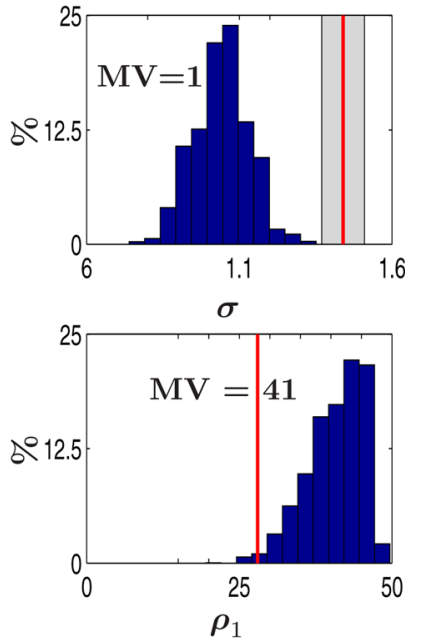

FIG. 8. (Color online) Posterior pdfs for the foam. Vertical lines and shaded area: reference values and standard deviations.
An evaluation of the marginal cost function for each parameter around the reference is presented for the three tested materials in Figs. 5-7. The effect of the prior information is more or less important depending on the material, but it still helps to regularize the identification problem for some parameters. Moreover, the minimum of the cost function is found to be very close to the expected values of the parameters in all cases.

The posterior pdfs obtained with the EMCMC method for the three tested materials are presented in Figs. 8, 9,

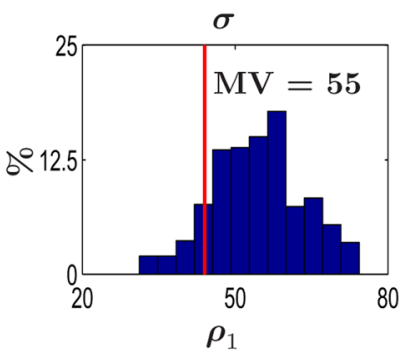

FIG. 9. (Color online) Posterior pdfs for the fibrous material. Vertical lines and shaded area: reference values and standard deviations. 

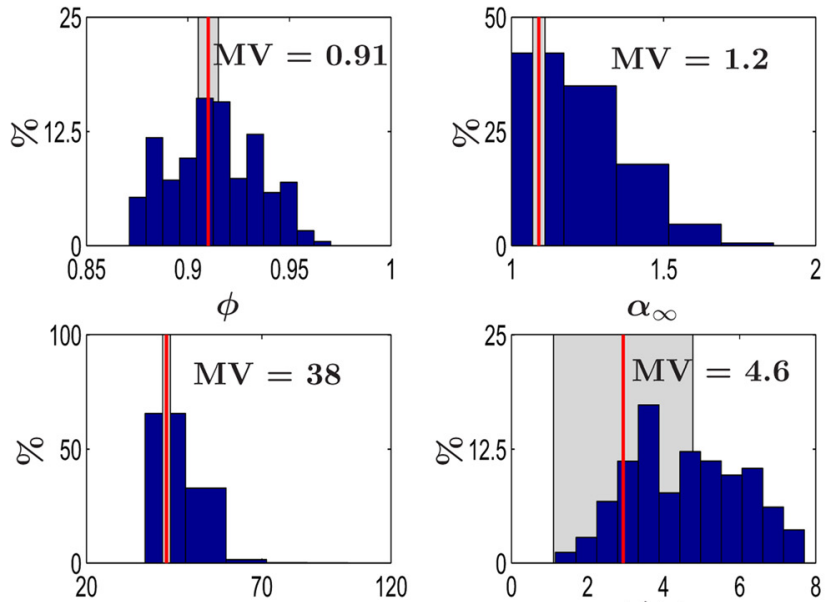

$\Lambda$

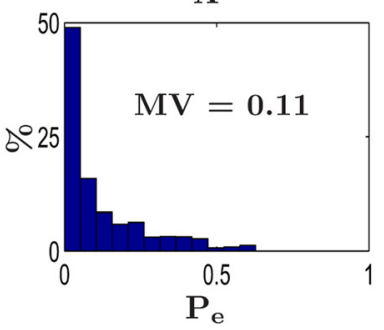

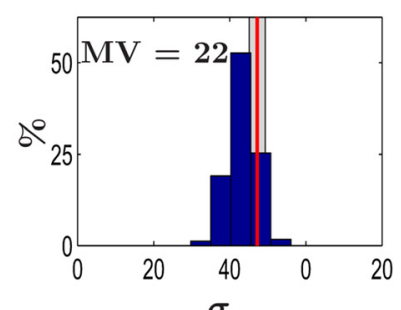

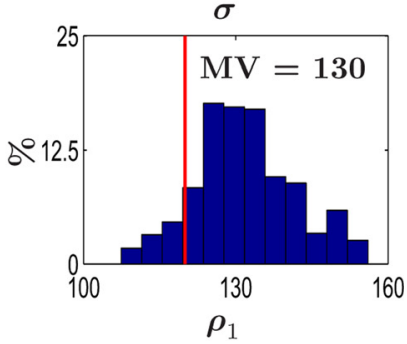

FIG. 10. (Color online) Posterior pdfs for the agglomerated material. Vertical lines and shaded area: reference values and standard deviations.

and 10, respectively. Very important information lies in the support of the posterior pdfs. The mean values, the maximum a posteriori, the standard deviations, and the joint probability distributions must be carefully analyzed to determine the relevance and the reliability of the results. Here, the distributions are quite smooth and unimodal, which means good reliability on the results. Nevertheless, some parameters, the solid phase parameters for instance, cannot be compared to reference values. The solid phase response is indeed not preponderant in the total response due to a very low rigidity, and the estimated values for the solid phase parameters may not be accurate.

The posterior mean values are now compared in Table VI with the reference values. Once again, the fibrous material with the lowest resistivity gives the best results in comparison with the indirect method. For the foam, some estimated values such as the tortuosity and the viscous characteristic length are slightly different from the reference values. However, the joint pdf in Fig. 11 is still very thin. These two parameters are thus still related. A better prior knowledge or a direct measurement on one of them could then modify the value of the second. For the agglomerated material, the thermal characteristic length is also different from its reference value. However, the posterior pdf on this

TABLE VI. Differences (\%) with the reference values.

\begin{tabular}{lccc}
\hline \hline Parameters & Foam & Fibrous & Agglomerated \\
\hline$\phi$ & 2.3 & 0.28 & 0.32 \\
$\alpha_{\infty}$ & 34 & 0.54 & 13 \\
$\sigma$ & 28 & 2.8 & 8.2 \\
$\Lambda$ & 32 & 1.8 & 15 \\
$\Lambda^{\prime}$ & 5.0 & 38 & 81 \\
$\rho_{1}$ & 46 & 23 & 9.8 \\
\hline \hline
\end{tabular}

parameter is very thick with a high standard deviation, and the reference value with its own uncertainties lies in the confidence interval.

The experimental uncertainties can indeed explain some differences. The indirect method in Refs. 18 and 19 and the present inverse method are both submitted to experimental uncertainties. For example, effects of circumferential edge constraint $^{16,42}$ can be expected in both measurements and can lead to differences in the results. The reference value of the resistivity obtained with a flowmeter is also submitted to similar uncertainties due to the boundary conditions. These uncertainties can thus change the accuracy of all the

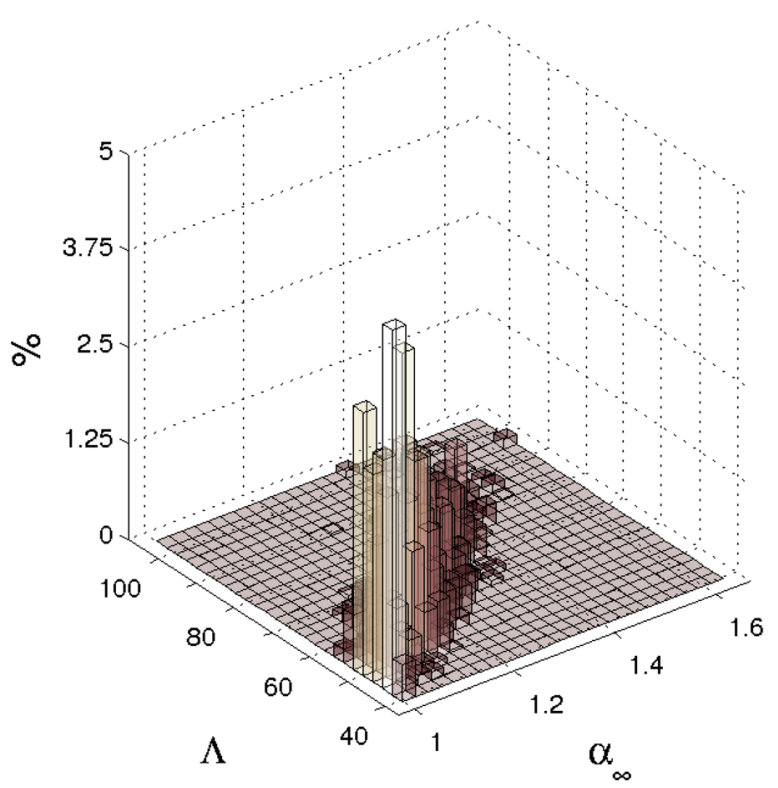

FIG. 11. (Color online) Joint pdf between the tortuosity and the viscous characteristic length for the foam. 
reference values. The advantage of the present method is to provide an idea of the interval of confidence and to identify the direct measurements that could improve the results.

It is also well known that measurement reproducibility and repeatability are not perfect, and round robin tests have already shown some surprising discrepancies between laboratories even for classical absorption coefficient measurements. The present comparison between results obtained in different laboratories, by different users, on different samples, with different methods seems therefore quite reasonable. A better prior knowledge on some parameters could improve the comparison, but the aim of the paper is not to present a perfect method that has been tuned to obtain the expected results. This paper presents instead a method and its limits, a method submitted to the user's expertise, and a flexible method that can be improved with external data obtained, for example, from direct measurements.

\section{CONCLUSION}

An inverse method of porous material identification has been tested on simulated and experimental data. This method based on Baye's rule is robust due to the prior information added in the cost function. The posterior pdfs are finally obtained with an EMCMC algorithm (genetic algorithm and Markov Chain Monte Carlo). Simulated and experimental results show that this method is efficient and more adapted to low resistivity materials. The posterior pdfs and the joint pdfs are also available in this method to evaluate the results accuracy and the dependence between the parameters. Compared to standard inverse methods, the main advantages are the robustness added by the prior information and the disposal of the full posterior pdfs rather than only point estimates.

Small discrepancies can be observed in a few cases with other characterization methods but some clues are given in the paper to help the user to evaluate the reliability of the results and, if necessary, to improve the quality of the results by adding prior information on some parameters with specific direct measurements. Once again, the main advantage of the method is not to measure the parameters with the highest reliability but to give, with a very simple measurement setup, the best set of parameters to feed a complex model being given all the possible modeling and measurement uncertainties, and to give also for each parameter an interval of confidence. It is then the user's responsibility to take the results as is or to investigate the possible enhancements.

\section{ACKNOWLEDGMENTS}

The authors acknowledge their industrial partners Matelys and Faurecia in this project. The authors also acknowledge the Project Pluri-Formations PILCAM2 at the Université de Technologie de Compiègne for providing HPC resources that have contributed to the research results reported within.

\section{APPENDIX: REDUCED ONE-DIMENSIONAL BIOT'S} MODEL

Using the wave formalism with a one-dimensional Biot's model gives the following wave numbers $k_{1}, k_{2}$ and amplitude coefficients $\mu_{1}, \mu_{2}$ between the solid and fluid displacements:

$$
\begin{aligned}
& k_{1}=\sqrt{\frac{\omega^{2}}{2\left(P R-Q^{2}\right)}\left(P \rho_{22}+R \rho_{11}-2 Q \rho_{12}-\sqrt{\Delta}\right)}, \\
& \mu_{1}=\frac{P k_{1}^{2}-\omega^{2} \rho_{11}}{\omega^{2} \rho_{12}-Q k_{1}^{2}}, \\
& k_{2}=\sqrt{\frac{\omega^{2}}{2\left(P R-Q^{2}\right)}\left(P \rho_{22}+R \rho_{11}-2 Q \rho_{12}+\sqrt{\Delta}\right)}, \\
& \mu_{2}=\frac{P k_{2}^{2}-\omega^{2} \rho_{11}}{\omega^{2} \rho_{12}-Q k_{2}^{2}},
\end{aligned}
$$

with

$$
\begin{aligned}
\Delta= & \left(P \rho_{22}+R \rho_{11}-2 Q \rho_{12}\right)^{2} \\
& -4\left(P R-Q^{2}\right)\left(\rho_{11} \rho_{22}-\rho_{12}^{2}\right) .
\end{aligned}
$$

${ }^{1}$ N. Atalla, R. Panneton, and P. Debergue, "A mixed displacement-pressure formulation for poroelastic materials," J. Acoust. Soc. Am. 104, 1444-1452 (1998).

${ }^{2}$ N. Atalla, M. A. Hamdi, and R. Panneton, "Enhanced weak integral formulation for the mixed (u,p) poroelastic equations," J. Acoust. Soc. Am. 109, 3065-3068 (2001).

${ }^{3} \mathrm{M}$. Biot, "Generalized theory of acoustic propagation in porous dissipative media.” J. Acoust. Soc. Am. 34, 168-178 (1962).

${ }^{4} \mathrm{M}$. Biot, "Theory of propagation of elastic waves in a fluid-saturated porous solid. I. Low-frequency range," J. Acoust. Soc. Am. 28, 168-178 (1956).

${ }^{5} \mathrm{M}$. Biot, "Theory of propagation of elastic waves in a fluid-saturated porous solid. I. Higher frequency range," J. Acoust. Soc. Am. 28, 179-191 (1956).

${ }^{6}$ Y. Champoux, M. R. Stinson, and G. A. Daigle, "Air-based system for the measurement of porosity," J. Acoust. Soc. Am. 89, 910-916 (1991).

${ }^{7}$ R. J. S. Brown, “Connection between formation factor for electrical resistivity and fluid-solid coupling factor in Biot equations for acoustic waves in fluid-filled media," Geophysics 45, 1269-1275 (1980).

${ }^{8}$ J. Allard, B. Castagnede, M. Henry, and W. Lauriks, "Evaluation of tortuosity in acoustic porous materials saturated by air," C. R. Acad. Sci. 322, 754-755 (1994)

${ }^{9}$ P. Leclaire, L. Kelders, W. Lauriks, M. Melon, N. Brown, and B. Castagnede, "Determination of the viscous and thermal characteristic lengths of plastic foams by ultrasonic measurements in helium and air," J. Appl. Phys. 80, 2009-2012 (1996).

${ }^{10}$ C. Ayrault, A. Moussatov, B. Castagnède, and D. Lafarge, "Ultrasonic characterization of plastic foams via measurements with static pressure variations,” Appl. Phys. Lett. 74, 2009-2012 (1999).

${ }^{11}$ R. L. Brown and R. H. Bolt, "The measurement of flow resistance of porous acoustic materials,” J. Acoust. Soc. Am. 13, 337-344 (1942).

${ }^{12}$ L. Jaouen, A. Renault, and M. Deverge, "Elastic and damping characterizations of acoustical porous materials: Available experimental methods and applications to a melamine foam," Appl. Acoust. 69, 1129-1140 (2008).

${ }^{13} \mathrm{~V}$. Tarnow, "Dynamic measurements of the elastic constants of glass wool,” J. Acoust. Soc. Am. 118, 3672-3678 (2005).

${ }^{14}$ C. Perrot, R. Panneton, and X. Olny, "Computation of the dynamic bulk modulus of acoustic foams," in Symposium on the Acoustics of PoroElastic Materials (SAPEM), Lyon (2005).

${ }^{15}$ C. Perrot, "Microstructure and acoustical macro-behavior: Approach by reconstruction of a representative elementary cell," J. Acoust. Soc. Am. 121, 2471-2471 (2007). 
${ }^{16}$ B. H. Song and J. S. Bolton, "A transfer-matrix approach for estimating the characteristic impedance and wave numbers of limp and rigid porous materials," J. Acoust. Soc. Am. 107, 1131-1152 (2000).

${ }^{17}$ N. Kino, T. Ueno, Y. Suzuki, and H. Makino, "Investigation of nonacoustical parameters of compressed melamine foam materials," Appl. Acoust. 70, 595-604 (2009).

${ }^{18} \mathrm{R}$. Panneton and X. Olny, "Acoustical determination of the parameters governing viscous dissipation in porous media," J. Acoust. Soc. Am. 119, 2027-2040 (2006).

${ }^{19} \mathrm{X}$. Olny and R. Panneton, "Acoustical determination of the parameters governing thermal dissipation in porous media," J. Acoust. Soc. Am. 123, 814-824 (2008).

${ }^{20}$ J.-P. Groby, E. Ogam, L. D. Ryck, N. Sebaa, and W. Lauriks, “Analytical method for the ultrasonic characterization of homogeneous rigid porous materials from transmitted and reflected coefficients," J. Acoust. Soc. Am. 127, 764-772 (2010).

${ }^{21}$ L. Boeckx, P. Leclaire, P. Khurana, C. Glorieux, W. Lauriks, and J. F. Allard, "Investigation of the phase velocities of guided acoustic waves in soft porous layers," J. Acoust. Soc. Am. 117, 545-554 (2005).

${ }^{22}$ L. Boeckx, P. Leclaire, P. Khurana, C. Glorieux, W. Lauriks, and J. F. Allard, "Guided elastic waves in porous materials saturated by air under Lamb conditions," J. Appl. Phys. 97, 094911 (2005).

${ }^{23}$ Y. Atalla, "Développement d'une technique inverse de caractérisation acoustique des matériaux poreux (Development of an inverse acoustical characterization technique for porous materials)," Thèse de l'Université de Sherbrooke, Québec, 2002, 212 pages.

${ }^{24}$ Y. Atalla and R. Panneton, "Inverse acoustical characterization of open cell porous media using impedance tube measurements," Can. Acoust. 33, 11-24 (2005)

${ }^{25}$ G. Iannace, C. Ianiello, L. Maffei, and R. Romano, "Characteristic impedance and complex wave-number of limestone chips," in Proceedings of the 4th European Conference on Noise Control - Euronoise (2001).

${ }^{26}$ T. Courtois, T. Falk, and C. Bertolini, "An acoustical inverse measurement system to determine intrinsic parameters of porous samples," in Symposium on the Acoustics of Poro-Elastic Materials (SAPEM), Lyon (2005).

${ }^{27}$ R. Dragonetti, C. Ianniello, and R. Romano, "The use of an optimization tool to search non-acoustic parameters of porous materials," in Proceedings of Inter-noise, Prague (2004).

${ }^{28}$ V. Gareton, D. Lafarge, and S. Sahraoui, "The measurement of the shear modulus of a porous polymer layer with two microphones," Polym. Test. 28, 508-510 (2009).
${ }^{29}$ Z. E. A. Fellah, C. Depollier, S. Berger, W. Lauriks, P. Trompette, and J.-Y. Chapelon, "Determination of transport parameters in air-saturated porous materials via reflected ultrasonic waves," J. Acoust. Soc. Am. 114, 2561-2569 (2003).

${ }^{30}$ Z. E. A. Fellah, M. Fellah, W. Lauriks, and C. Depollier, "Direct and inverse scattering of transient acoustic waves by a slab of rigid porous material," J. Acoust. Soc. Am. 113, 61-72 (2003).

${ }^{31}$ Z. Fellah, F. Mitri, M. Fellah, E. Ogam, and C. Depollier, "Ultrasonic characterization of porous absorbing materials: Inverse problem," J. Sound Vib. 302, 746-759 (2007)

${ }^{32}$ E. Ogam, Z. Fellah, N. Sebaa, and J.-P. Groby, "Non-ambiguous recovery of Biot poroelastic parameters of cellular panels using ultrasonic waves." J. Sound Vib. 330, 1074-1090 (2011).

${ }^{33}$ A. Tarantola, Inverse Problem Theory and Methods for Model Parameter Etimation (Society for Industrial and Applied Mathematics, Philadelphia, 2005), pp. 1-352.

${ }^{34} \mathrm{C}$. Robert, The Bayesian Choice: From Decision-Theoretic Foundations to Computational Implementation, 2nd ed. (Springer, New York, 2001), Chap. 1, p. 10.

${ }^{35}$ D. R. Brillinger, Time Series: Data Analysis and Theory (Society for Industrial and Applied Mathematics, Philadelphia, 2001), pp. 1-540.

${ }^{36}$ W. Gilks, S. Richardson, and D. Spiegelhalter, Markov Chain Monte Carlo in Practice (Chapman and Hall, London, 1995), pp. 1-486.

${ }^{37}$ W. Hastings, "Monte Carlo sampling methods using Markov chains and their applications," Biometrika 57, 97-109 (1970).

${ }^{38}$ F. Liang and W. Wong, "Real-parameter evolutionary Monte Carlo with applications to Bayesian mixture models," J. Am. Stat. Assoc. 96, 653-666 (2001).

${ }^{39}$ B. Zhang and D. Cho, "System identification using evolutionary Markov chain Monte Carlo,” J. Syst. Archit. 47, 587-599 (2001).

${ }^{40} \mathrm{~B}$. Hu and K. Tsui, "Distributed evolutionary Monte Carlo for Bayesian computing," Comput. Stat. Data Anal. 54, 688-697 (2010).

${ }^{41}$ O. Doutres, N. Dauchez, J. Gnevaux, and O. Dazel, "Validity of the limp model for porous materials: A criterion based on the Biot theory," J. Acoust. Soc. Am. 122, 2038-2048 (2007).

${ }^{42}$ K. V. Horoshenkov, A. Khan, F.-X. Bécot, L. Jaouen, F. Sgard, A. Renault, N. Amirouche, F. Pompoli, N. Prodi, P. Bonfiglio, G. Pispola, F. Asdrubali, J. Hübelt, N. Atalla, C. K. Amédin, W. Lauriks, and L. Boeckx, "Reproducibility experiments on measuring acoustical properties of rigidframe porous media (round-robin tests)," J. Acoust. Soc. Am. 122, 345-353 (2007) 
Copyright of Journal of the Acoustical Society of America is the property of American Institute of Physics and its content may not be copied or emailed to multiple sites or posted to a listserv without the copyright holder's express written permission. However, users may print, download, or email articles for individual use. 